\title{
The genetic and molecular origin of natural variation for the fragrance trait in an elite Malaysian aromatic rice through quantitative trait loci mapping using SSR and gene-based markers
}

\author{
Farahnaz Sadat Golestan Hashemi ${ }^{\text {a }}$, Mohd Y. Rafii ${ }^{\mathrm{a}, \mathrm{b}, *}$, Mohd Razi Ismail ${ }^{\mathrm{a}, \mathrm{b}}$, \\ Mahmud Tengku Muda Mohamed ${ }^{b}$, Harun A. Rahim ${ }^{c}$, Mohammad Abdul Latif ${ }^{\text {a,d }}$, Farzad Aslani ${ }^{\text {b }}$ \\ a Institute of Tropical Agriculture, Universiti Putra Malaysia, 43400 UPM Serdang, Selangor, Malaysia \\ b Department of Crop Science, Faculty of Agriculture, Universiti Putra Malaysia, 43400 UPM Serdang, Selangor, Malaysia \\ c Bioscience and Agrotechnology Division, Malaysian Nuclear Agency, Bangi, 43000 Kajang, Selangor, Malaysia \\ d Bangladesh Rice Research Institute (BRRI), Gazipur 1701, Bangladesh
}

\section{A R T I C L E I N F O}

\section{Article history:}

Received 16 July 2014

Received in revised form 9 October 2014

Accepted 27 October 2014

Available online 29 October 2014

\section{Keywords:}

Fragrant rice

QTL

2-Acetyl-1-pyrroline

GC-MS

Microsatellite marker

Gene-specific marker

\begin{abstract}
A B S T R A C T
MRQ74, a popular aromatic Malaysian landrace, allows for charging considerably higher prices than nonaromatic landraces. Thus, breeding this profitable trait has become a priority for Malaysian rice breeding. Despite many studies on aroma genetics, ambiguities considering its genetic basis remain. It has been observed that identifying quantitative trait loci (QTLs) based on anchor markers, particularly candidate genes controlling a trait of interest, can increase the power of QTL detection. Hence, this study aimed to locate QTLs that influence natural variations in rice scent using microsatellites and candidate gene-based sequence polymorphisms. For this purpose, an $\mathrm{F}_{2}$ mapping population including 189 individual plants was developed by MRQ74 crosses with 'MR84', a non-scented Malaysian accession. Additionally, qualitative and quantitative approaches were applied to obtain a phenotype data framework. Consequently, we identified two QTLs on chromosomes 4 and 8. These QTLs explained from $3.2 \%$ to $39.3 \%$ of the total fragrance phenotypic variance. In addition, we could resolve linkage group 8 by adding six gene-based primers in the interval harboring the most robust QTL. Hence, we could locate a putative $f g r$ allele in the QTL found on chromosome 8 in the interval RM223-SCU015RM (1.63 cM). The identified QTLs represent an important step toward recognition of the rice flavor genetic control mechanism. In addition, this identification will likely accelerate the progress of the use of molecular markers for gene isolation, genebased cloning, and marker-assisted selection breeding programs aimed at improving rice cultivars.
\end{abstract}

(c) 2014 Elsevier B.V. All rights reserved.

\section{Introduction}

Rice is the most significantly consumed grain for human nutrition and caloric intake worldwide, particularly in Asia. Rice is mostly bred for crop quality and productivity, and there are varieties selected for traits such as smell, texture and firmness (Childs and Burdett, 2000).

Abbreviations: QTLs, quantitative trait loci; MAS, marker assisted selection; $2 \mathrm{AP}$ 2-acetyl-1-pyrroline; LOD, logarithm of odds; DNA, deoxyribonucleic acid; CTAB cetyltrimethylammonium bromide; PCR, polymerase chain reaction; dNTPs, deoxyribonucleotide triphosphate; RM, rice microsatellites; SSR, simple sequence repeats; SPME, solid-phase microextraction; GC/MS, gas chromatography/mass spectrometry; NIST, National Institute of Standards and Technology; TAE, Tris-acetate-EDTA; BADH, betaine aldehyde dehydrogenase; SNP, single nucleotide polymorphism; MARDI, Malaysian Agriculture Research and Development Institute; UPM, Universiti Putra Malaysia; HK, Haley-Knott regression; EHK, extended Haley-Knott regression; CIM, composite interval mapping; MI, multiple imputation; SDR, segregation distorted region; SDL, segregation distortion loci.

* Corresponding author at: Institute of Tropical Agriculture, Universiti Putra Malaysia, 43400 UPM Serdang, Selangor, Malaysia.

E-mail addresses: fsgolestan@yahoo.ca (F.S. Golestan Hashemi), mrafii@upm.edu.my (M.Y. Rafii).
Quality is regarded as a significant factor in rice production and marketing. The inherent rice quality is of great importance because the majority of rice produced is consumed as a kernel, whereas the percentage of rice processed into flakes is small (Huang et al., 1998).

Aroma is considered one of the most worthwhile traits for rice grain quality. Strong fragrance expression plays a significant role in rice marketing. Rice aroma has been popular among consumers worldwide, particularly in the Middle East and Western communities. Among the more than 100 volatile flavor compounds associated with aromatic rice, 2acetyl-1-pyrroline (2AP) has been found to be the primary reason for the distinctive aroma in Basmati and Jasmine rice. While 2AP is known as the main aromatic compound in fragrant rice, a range of volatile components provides every variety with its own unique aroma. However, little is known about the contribution of these volatiles to fragrance (Golestan Hashemi et al., 2013).

Although various significant traits are controlled by loci that are effective on phenotypes, many agronomically valuable characteristics such as quality, yield, and tolerance for abiotic stresses, are naturally quantitative. The genes controlling such characteristics are polygenes 
or minor genes that, although show Mendelian inheritance, are highly affected by the environment. The emergence and availability of molecular markers and whole genome sequences of rice have opened new windows for identifying and mapping quantitative trait loci (QTLs) that confer the aroma trait. In addition, it is of great significance to use molecular markers for identifying QTLs from unadapted germplasm or wild species that are capable of developing rice grain yields (Khush et al., 2001). Genetic analysis has indicated that the aromatic trait is governed by recessive monogenic inheritance, which is independent of cytoplasmic genes. However, fragrance has also been investigated as a quantitative characteristic, and several genes are involved in its expression in rice (Hien et al., 2006). Such opposing observations have revealed the complexity of the genetic inheritance of aroma and the location of underlying gene(s) in rice (Chaut et al., 2010; Sakthivel et al., 2009b).

Through QTL mapping, fine mapping, sequence analysis, and complementation testing, the badh2 locus in rice comprising the fgr gene has been known to be a main genetic aroma determinant in all aromatic rice lines (Kovach et al., 2009). The fgr gene on chromosome 8 encodes the betaine aldehyde dehydrogenase (BADH) enzyme (Bradbury et al., 2005a; Chen et al., 2006). Moreover, two null aromatic recessive alleles have been detected in rice (Shi et al., 2008): badh2-E7 (involving Basmati and Jasmine sorts) (Kovach et al., 2009) is an 8 bp deletion and 3 single nucleotide polymorphisms (SNPs) in the 7th exon (Saha et al., 2012) and badh2-E2 is a sequence identical to the badh2 allele but includes a 7 bp deletion in the 2 nd exon (Shi et al., 2008). Both null badh2 alleles associate with rice flavor. Thus far, no difference has been detected between these two null badh2 alleles in relation to generating rice scent and affecting its yield. Therefore, it appears to be feasible that both can be used in breeding for scented rice varieties (Shi et al., 2008).

However, $B A D H 2$ is not the only gene governing the aroma trait in rice because (1) some varieties are conditioned by only one dominant locus but involve the deletion of BADH2 (Bradbury et al., 2005a), (2) elimination of the $B A D H 2$ gene is not common in all rice varieties with the aroma trait as such elimination has never been observed in some aromatic rice cultivars (Sakthivel et al., 2009b), and (3) different varieties of fragrant rice have different mechanisms (Kuo et al., 2005). In addition, it has been hypothesized that the pleasant flavor of some varieties might be governed by other genes and not badh2 (Sakthivel et al., 2009a), as many genotypes have unknown eliminations and involved a negligible amount of 2AP including higher levels of other aromatic components such as 4-vinyl-2-methoxy phenol and benzyl alcohol. For example, $B A D H 1$, a homolog of BADH2 (Os04 g39020; 92\% homology), has been identified on rice chromosome 4 as a potential candidate gene as an aroma QTL (Bradbury et al., 2005a).

Although a single recessive gene for aroma has been detected, different studies have observed QTLs controlling aroma and the inherent nature (dominant or recessive) of this trait (Amarawathi et al., 2008; Chaut et al., 2010; Lorieux et al., 1996). Thus, the genetic basis of rice scent has become a complicated issue. Such opposing views reveal that neither 2AP nor badh2 alone is responsible for aroma and show that the contribution and existence of other gene(s) or loci and volatile compounds affect the aroma trait (Golestan Hashemi et al., 2013). Hence, a segregating population of $\mathrm{F}_{2}$ individuals was generated in a two-year effort to explore associations between the fragrance phenotype and particular genetic loci in charge of its variation using published microsatellites and candidate gene-specific markers. Identification of the linkage between scent and candidate gene loci using co-dominant markers might increase the power of QTL detection.

\section{Materials and methods}

\subsection{Plant material}

An $F_{2}$ population was used for QTL analysis. This population was obtained from a cross of the rice parental inbred line MR84, a non-fragrant Malaysian cultivar, and MRQ74, a fragrant Malaysian rice cultivar, in
2011-2013. A single $F_{1}$ plant with markers confirming the hybridity of the cross MR8 $4 \times$ MRQ74 was chosen for generating an $F_{2}$ population in the Malaysian Agriculture Research and Development Institute (MARDI). Then, $189 \mathrm{~F}_{2}$ individuals were grown in a rice field at Universiti Putra Malaysia (UPM). $F_{3}$ self-pollinated seeds from each line were harvested, dried under ambient temperature, and threshed with minimal exposure to direct sunlight.

\subsection{Sensory and quantitative analytical approaches for fragrance assessment}

\subsubsection{Chemical method}

When the plants were at the tillering stage, $1 \mathrm{~g}$ of leaves was excised, cut into small pieces and incubated with $10 \mathrm{ml} \mathrm{1.7 \%} \mathrm{KOH} \mathrm{in} \mathrm{a} \mathrm{Petri} \mathrm{dish}$ at room temperature. After 15 min of incubation, the samples were opened one by one, and the odor was smelled by a trained threemember panel. Assessments were randomly performed to avoid desensitization. Additionally, the assessment of each sample was performed in triplicate. Aroma was rated based on the fragrance intensity ranging from zero to three, corresponding to the absence of fragrance, faint fragrance, moderate fragrance, and strong fragrance.

\subsubsection{SPME/GC-MS}

Solid-phase microextraction (SPME) connected to gas chromatography/mass spectrometry (GC/MS) (Thermo Scientific, TSQ Quantum XLS, USA) was used to discern aromatic from non-aromatic rice kernels and then to quantify 2AP in aromatic individuals by comparing their mass spectra with that in the National Institute of Standards and Technology (NIST, ver. 2.0f, 2008) mass spectral database. Because of the difficulty and expense of the 2AP analysis, this experiment was performed without replication. We used GC with a TG-5MS capillary column ( $30 \mathrm{~m} \times 0.25 \mu \mathrm{m}$ ) and MASS to analyze headspace volatiles (Thermo Scientific, TSQ Quantum XLS, USA). Volatiles were derived and concentrated using a pre-conditioned (at $250{ }^{\circ} \mathrm{C}$ for $30 \mathrm{~min}$ ) SPME fiber connected to a SPME autosampler holder (57284-U) (Supelco, Bellefonte, PA,

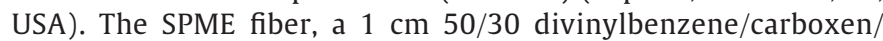
polydimethylsiloxane stableflex fiber (carboxen/DVB/PDMS) (Supelco, Bellefonte, PA, USA), was desorbed for $5 \mathrm{~min}$ in a GC injector at $250{ }^{\circ} \mathrm{C}$ in splitless mode. The GC oven program was held for 1 min at $50{ }^{\circ} \mathrm{C}$ and then increased to $100{ }^{\circ} \mathrm{C}$ at a rate of $4{ }^{\circ} \mathrm{C} / \mathrm{min}$ and further increased to $240{ }^{\circ} \mathrm{C}$ at $50{ }^{\circ} \mathrm{C} / \mathrm{min}$ with a final hold of $2 \mathrm{~min}$. The extraction was performed in $20 \mathrm{ml}$ screw top vials $(75.5 \times 22.5 \mathrm{~mm})$ with an $18 \mathrm{~mm}$ screw cap with septa (Agilent Technologies, Germany). The vials were heated in an oven set to $150{ }^{\circ} \mathrm{C}$ for $1 \mathrm{~h}$ prior to the removal of unintended volatile compounds.

\subsection{DNA and marker analysis}

Fresh leaves were harvested from single plants and frozen in liquid nitrogen. For genotyping, high-quality genomic deoxyribonucleic acid (DNA) was isolated from $20-25 \mathrm{mg}$ of dried $\mathrm{F}_{2}$ leaves according to the cetyltrimethylammonium bromide (CTAB) approach (Gawel and Jarret, 1991 ) and diluted to a concentration of ca. $70 \mathrm{ng} / \mathrm{l}$ for marker analysis. Based on previous studies, a rice molecular linkage map, and the published microsatellite database by Temnykh et al. (2000) and McCouch et al. (2002) (http://www.gramene.org/microsat), 512 pairs of Simple Sequence Repeat (SSR) primers (First BASE Laboratories Sdn Bhd Co., Ltd, Malaysia) and six gene-based markers (Table 1) that cover the whole rice genome were combined to perform a comparative polymorphic analysis of the two parents and two randomly selected $F_{1}$ progenies with 108 confirmed to be polymorphic when assessing the segregation ratios of the $\mathrm{F}_{2}$ progenies. For all progenies, the loci were expanded in PCR reactions in total volumes of $15 \mu \mathrm{l}$ including genomic DNA as template, $0.1 \mu \mathrm{M}$ forward and reverse primers, $80 \mu \mathrm{M}$ dNTPs, $2 \mathrm{mM} \mathrm{MgCl}_{2}$ and $0.5 \mathrm{U}$ Taq DNA polymerase in a T100-Thermal Cycler (BIO-RAD, USA). A touchdown PCR protocol was performed as described via Bradbury et al. (2005b). Afterward, differences in limitations in banding patterns were resolved using gel 
Table 1

Nomenclature of SSR and gene-specific markers.

\begin{tabular}{|c|c|c|c|c|}
\hline \multirow[t]{2}{*}{ Locus } & \multirow[t]{2}{*}{ Primers $\left(5^{\prime}-3^{\prime}\right)$} & \multicolumn{2}{|c|}{ Nature of polymorphism } & \multirow[t]{2}{*}{ References } \\
\hline & & MR84 & MRQ74 & \\
\hline \multirow[t]{2}{*}{ L06 } & GCAAGTGACGGAGTACGCCT & 348 bp & $391 \mathrm{bp}$ & Chen et al., (2006) \\
\hline & GCTAACTTCCGCTCACGCAA & & & \\
\hline \multirow[t]{2}{*}{ NKSbad2 } & GGTTGCATTTACTGGGAGTTATG & 90 bp & 82 bp & Amarawathi et al., (2008) \\
\hline & CAAACAAAGGTTTAAAGACACCT & & & \\
\hline \multirow[t]{2}{*}{ FMbadh2-E7 } & GGTTGCATTTACTGGGAGTT & 268 bp & $260 \mathrm{bp}$ & Shi et al., (2008) \\
\hline & CAGTGAAACAGGCTGTCAAG & & & \\
\hline \multirow[t]{2}{*}{ BADEX7-5 } & TGTTTTCTGTTAGGTTGCATT & 103 bp & $95 \mathrm{bp}$ & Sakthivel et al., (2009a) \\
\hline & ATCCACAGAAATTTGGAAAC & & & \\
\hline \multirow[t]{2}{*}{ Aro7 } & ATTTGCCTCCTGAGTCTG & 302 bp & - & Sun et al., (2008) \\
\hline & GAGGATGGGGAAGATAAA & & & \\
\hline \multirow[t]{2}{*}{ SCU015RM } & GGTTCATTCAAGCCTCCAGC & - & - & Cordeiro et al., (2002) \\
\hline & TTTTCCCACCAGCCAAACAT & & & \\
\hline
\end{tabular}

electrophoresis in a $3 \%$ MetaPhore gel in $0.5 \times$ Tris-acetate-EDTA (TAE) buffer together with known concentrations of genomic DNA ladder as a standard.

\subsection{Statistical and QTL analyses}

The qualitative and quantitative distribution of the trait was examined for 189 individuals. Standard deviation and mean were also calculated. Deviations from a Mendelian ratio were tested by the Chi-square goodness of fit test $(P<0.05)$, and the analyses were performed using $\mathrm{R}$ statistical software (R Core Team, 2011).

To construct the genetic linkage map, polymorphic SSR markers were converted into genotype codes according to the scores of the parents. For quality filtering, pre-selection with regard to genotyping errors and segregation ratios was performed as markers remarkably $(P<1 \mathrm{e}-10)$ deviated from the expected 1:2:1 ratio in the Chi-square test, and a genotyping error LOD $=2$ would be excluded from further examination. Linkage analyses were performed using the R/qtl package (Broman et al., 2003). Polymorphic SSR markers were initially classified. Then, each group was compared to the Rice Genome Database (http://www.gramene.org) sequence and edited for SSR position. Afterward, each group was separately regenerated using a minimum 6.0 log of odds (LOD) and a 0.35 recombination frequency maximum. In each group, the plotting of marker order was achieved using "plot.rf". The final linkage map was created using the "ripple" function $(P<0.005)$. Marker orders conflicting with the physical map were recalculated according to LOD rescores by the “."switch.order" function" in R/qtl. Kosambi's mapping function was used to calculate the map distances (Kosambi, 1943). The accuracy of the linkage map was investigated and verified by calculating the pairwise resynthesis of fractions across the genome and comparing the marker order with the physical location in the rice genome. Additionally, the RCircos package was used to create a graphical view of the entire linkage groups in this study (Zhang et al., 2013).

All phenotype and genotype data were analyzed to detect the maineffects of the QTLs with R/qtl software (Broman et al., 2003). Significant thresholds were extracted from 1000 permutations based on a genomewide type I error rate of 5\% for identifying a QTL as important. First, the "calc.genoprob" function in R/qtl was used to examine the potential of true underlying genotypes given the observed marker data. Second, multiple imputation (MI), Haley-Knott regression (HK), extended Haley-Knott regression (EHK), composite interval mapping (CIM) and multiple mapping approaches were compared to show the most powerful method for identifying chromosomal areas related to aroma. The MI method differs in that it guesses, or imputes, genotypes between markers for every individual given marker locations, marker scores and the probability of recombination events between markers for a given population type, whereas the HK approach provides a faster approximation for identifying genotype data. CIM was executed by selecting tree markers as cofactors to remove biases that may be caused by QTLs associated with the position tested. A LOD score $>3.3$ extracted from 1000 permutations at a $5 \%$ important level was used to identify QTLs to minimize type II errors. Additionally, one-dimensional scans were used to identify putative locations of additive QTLs. In addition, two-dimensional scans within a twoQTL model were performed with the thresholds identified with 1000 permutations at a 5\% significance level. For each QTL location, the $95 \%$ Bayesian confidence interval was estimated. The confidence interval was expanded to the nearest flanking markers. The dominant and additive impact and percentage of phenotypic variance described via each QTL $\left(\mathrm{R}^{2}\right)$ at the maximum LOD score were calculated using the makeqtl and fitqtl functions in R/qtl. For the nomenclature of the detected QTLs, we first used a three-letter abbreviation for the fragrance trait followed by the chromosome on which the QTL was found (McCouch, 2008). We used a rice genetic linkage map, the Cornell SSR map, to compare the QTL locations found in this study.

\section{Results}

\subsection{Trait performance}

Comparing the aroma of the parental lines verified the fragrance in 'MRQ74' with $169 \pm 4.8 \mathrm{ppb} 2 \mathrm{AP}$ (average of six evaluations), while 'MR84' had no 2AP. The distribution of the 2AP values in the segregating population was between the values of the parental lines (ranging from 9 to $179 \mathrm{ppb}$ ) without notable transgressive segregation (Fig. 1). Only a small proportion of the $\mathrm{F}_{2}$ individuals could reconstitute the original MRQ74 fragrance, suggesting the engagement of many genes. We achieved good correlated results $(r=0.908)$ via aroma tests using chemical (Mean $=1.2$, Std. Deviation $=1.043$ ) and SPME/GC-MS $($ Mean $=65.94$, Std. Deviation $=63.25)$ approaches with the exception of some cases. The trait showed no continuous variation with normal distribution. There was phenotypic skewness toward the non-scented parent (MR84). The Kolmogorov-Smirnov normality test indicated a $P<0.01$ for the aroma characteristic.

\subsection{Molecular marker analysis}

Out of 512 SSR and six gene-based markers covering the whole rice genome, 108 (20.8\%) demonstrated a polymorphism between the parental lines. The characteristics of the gene-based markers used are shown in Table 1. These candidate gene markers were obtained from the homologous sequences for the fgr gene involved in 2AP metabolism. Analysis of marker loci segregation patterns revealed that the majority of the marker loci examined had expected Mendelian segregation $(1: 2: 1)$ in the $F_{2}$ population. Among these primers, 88 (81.5\%) had obvious segregating loci. However, segregation distortion was found for 20 (18.5\%) marker loci that were distributed across 9 chromosomes with the exception of chromosomes 2, 8 and 11. Among these 20 markers, 11 (55\%) and 9 (45\%) were sloped toward MR84 and MRQ74, respectively. 

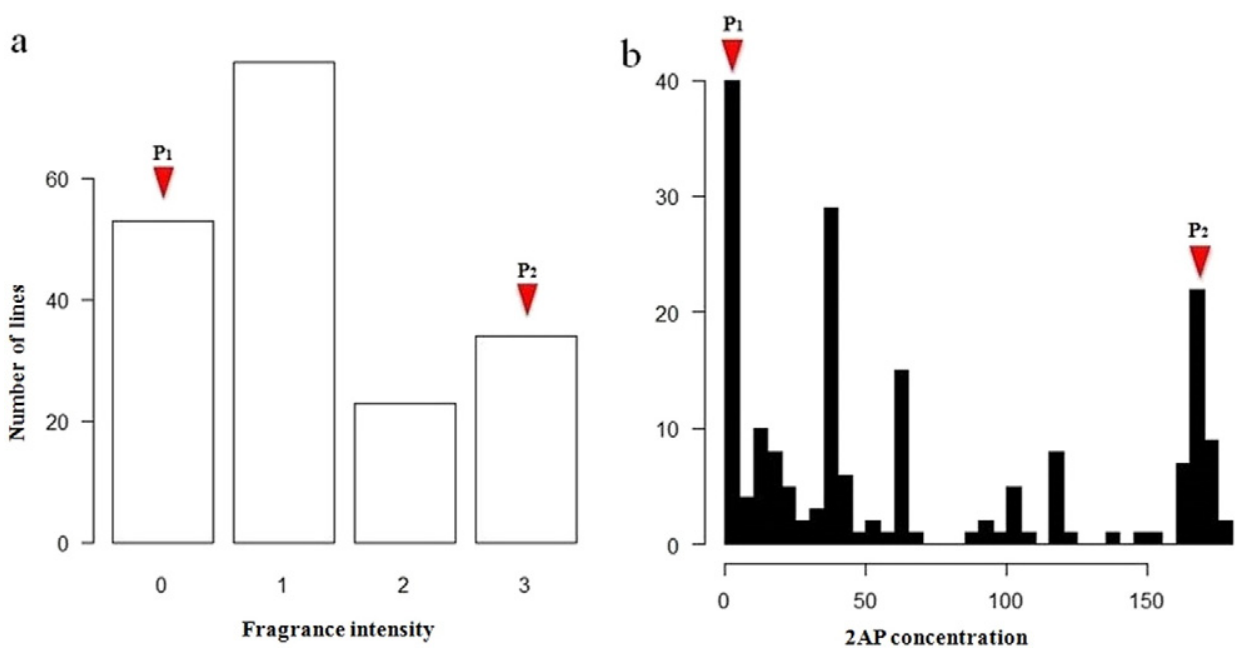

Fig. 1. Distribution of the fragrance in the $F_{2}$ population derived from MR84 $\left(P_{1}\right) \times \operatorname{MRQ74}\left(P_{2}\right)$ using qualitative (a) and quantitative (ppb) methods (b).

A segregation distorted region (SDR) containing 13 markers was detected on chromosomes 3, 4, 5, 6 and 7 in the same areas in which gametophytic or sterility loci (ga/S) were observed.

\subsection{Detection of QTLs for fragrance}

Composite interval analysis was approximately in agreement with the results obtained from the interval and multiple mapping approaches with respect to the likely position of the QTL that influenced fragrance. However, the most powerful approach for the population was CIM with a step interval of 1.0 based on visual inspection of one-dimensional LOD profile plots. Hence, in this study, putative QTL regions related to the odor trait were mapped by composite interval mapping with a 1000-permutation test. The experimental threshold LOD mean was 3.3 at a $5 \%$ level of importance. The linkage map, comprising 108 markers, could almost cover the entire rice genome (Fig. 2). The total coverage of the map was $1959.03 \mathrm{cM}$ (centiMorgans), and the average marker distance was $21.73 \mathrm{cM}$. According to the LOD threshold, two QTLs for fragrance were found on chromosomes 4 and 8, designated frg8-1 and frg4-1, respectively (Fig. 2). The putative QTL with a LOD score of 22.83 was initially located in the interval between RM223-RM515 $(2.34 \mathrm{cM})$ on the long arm of the 8th rice chromosome and highly contributed to the phenotypic variance, describing $39.3 \%$ of the total (Table 2 ). To positionally clone the fragrance gene, a fine-scale map with great density primers between the flanking markers, RM223 (20.65 Mb) and RM515 (20.28 Mb), was needed. Hence, six polymorphic gene-based markers were added to the area surrounding the LOD support interval for the identified QTL to resolve and saturate the linkage gap. Finally, as a critical region, the $1.6 \mathrm{cM}$ map interval between RM223 and SCU015RM was found to be significant for map-based cloning (Fig. 2). Another effective QTL was found at RM5633 in the SSR marker interval RM335-RM273 on chromosome 4 with a LOD score of 6.08, describing $3.2 \%$ of the phenotypic variation (Fig. 2; Table 2). At these QTLs, the 'MRQ74' allele could increase aroma because the mean 2AP of the lines homozygous for the 'MRQ74' allele at the markers close to the detected QTLs was remarkably higher than those of the homozygous lines for the 'MR84' allele (Fig. 1). Additionally, identifying any epistatic interaction between the QTLs required a two-dimensional scan that did not reveal important epistatic interactions in the entire genome in this population (Fig. 3).

\section{Discussion and conclusions}

Aromatic rice is a desirable product for humans as its market value is increasing. Although fragrance is regarded as an important trait of different breeding programs (Golestan Hashemi et al., 2013), few studies have investigated the biochemical pathway of rice fragrance, and some ambiguities with regard to the genetic basis of aroma remain. Hence, detecting the most significant genes underlying the aroma characteristic is complicated. Moreover, fragrance assessment in rice is difficult and traditional approaches e.g., smelling or chewing, are not reliable due to their subjectivity (Lorieux et al., 1996). Mapping grain fragrance QTLs is considered a qualitative trait based on sensory evaluations. However, volatile compounds of various fragrant rice cultivars, particularly 2AP, varied quantitatively (Hien et al., 2006; Vanavichit and Yoshihashi, 2010). Hence, to achieve a quantitative and unambiguous assessment of rice odor, 2AP levels should be directly examined using a more sensitive approach such as GC-MS (Chen et al., 2006). This method can detect the presence of 2AP at the ppb level with good repeatability (Lorieux et al., 1996). Additionally, without an accurate quantitative measurement of fragrance, transgressive segregation appears to be impossible (Amarawathi et al., 2008). For instance, Amarawathi et al. (2008) were unable to rule out the possibility of transgressive segregation due to lack of precise quantitative aroma evaluation. Knowledge of the occurrence of transgressive segregation will assist us in exploring the presence of modifying genes. In such cases, it is possible to improve these traits via recombination breeding (Amarawathi et al., 2008). Hence, the approach applied here was gas chromatographic quantification of volatile 2AP in rice grains together with a leaf sensory assay. Although the results of the chemical test correlated well with the results obtained by GC-MS, supporting the reliability of the sensory test for early selection in the MAS program, GC-MS was a more sensitive and accurate technique because it obtained unambiguous data for all of the lines tested (Lorieux et al., 1996). For further exploration of the novel fragrance gene(s) in MRQ74, quantification of the 2AP content in segregating progenies is necessary (Pinson, 1994).

To determine the loci involved in the variation for a particular phenotype, the placement of candidate genes and QTLs on linkage maps for seeking coincidence in a map position is regarded a promising method for several economic plants (Jeennor and Volkaert, 2014). Because no molecular research has been carried out on the genetic and inheritance basis of aroma for the Malaysian rice genetic pool, the goal of this research was to navigate the genetic principles of the inheritance of aroma in an elite Malaysian fragrance rice accession, MRQ74, with an appealing popcorn-like smell, and the location of the underlying gene(s) based on precise measurement of $2 \mathrm{AP}$. QTL mapping was constructed in the $\mathrm{F}_{2}$ mapping population derived from crosses with 'MR84', a high yielding non-aroma Malaysian accession.

Here, gene-based markers merged with SSR markers were used to establish marker-QTL linkage for fragrance in the population. The QTL 


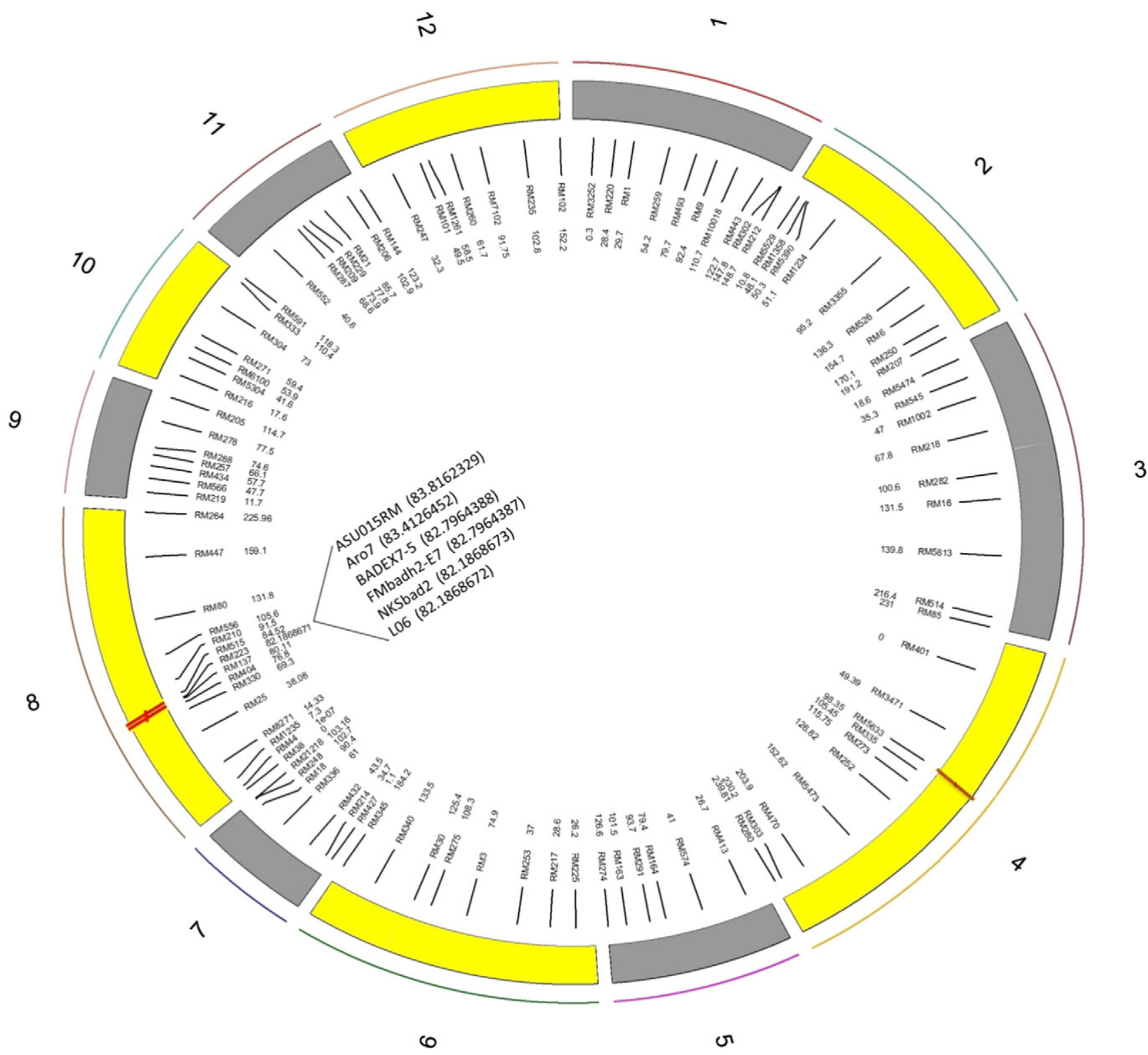

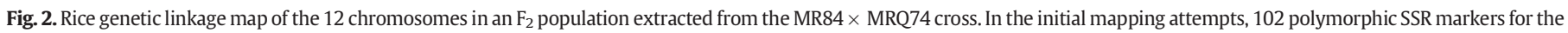

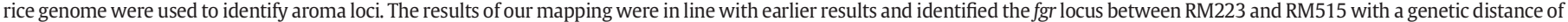

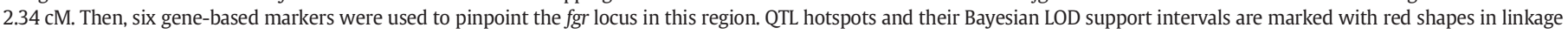

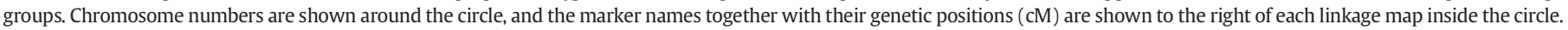

identified on chromosome 8 with the highest impact indicated a high LOD value and strong association with the total phenotypic variance (Fig. 2; Table 2). The physical position of the markers highly linked to the LOD peak in this QTL showed that they correspond to the same locus. Hence, it appears that the fgr locus, which indicates rice fragrance, is on the long arm of chromosome 8 between the SSR markers RM223 and RM515 (2.34 cM). The co-localization of candidate gene markers with the putative QTL shows that its gene might be responsible for a portion of the aroma variation found in this study. Several studies, including Bradbury et al. (2005a) and Chen et al. (2006), utilizing rice genome sequence information (IRGSP 2005) have determined that badh2 is a candidate gene for fragrance on chromosome 8 , and it encodes the

Table 2

Detected putative QTLs for fragrance in the $\mathrm{F}_{2}$ population.

\begin{tabular}{|c|c|c|c|c|c|c|c|c|c|c|}
\hline Chr. & QTL name & Nearest marker & $\mathrm{cM}$ & Marker interval & NLM (cM) & NRM (cM) & LOD & $\mathrm{AE}$ & $\mathrm{DE}$ & $\mathrm{R}^{2}$ \\
\hline 4 & frg $4-1$ & RM5633 & 0.0 & RM335-RM273 & 7.1 & 10.3 & 6.1 & 3.4 & -0.7 & 3.2 \\
\hline 8 & frg8-1 & NKSbad2 & 0.0 & RM223-SCU015RM & 0.0000002 & 1.62 & 22.83 & 12 & -1.9 & $\begin{array}{l}39.3 \\
(51.4)\end{array}$ \\
\hline
\end{tabular}

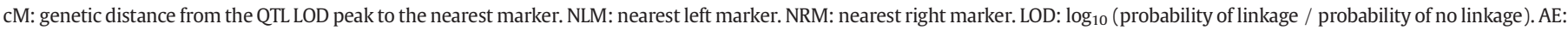

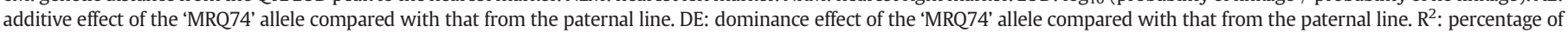
phenotypic variance explained by each QTL. Numbers in parentheses indicate the total percentage of phenotypic variance explained by multiple QTLs. 


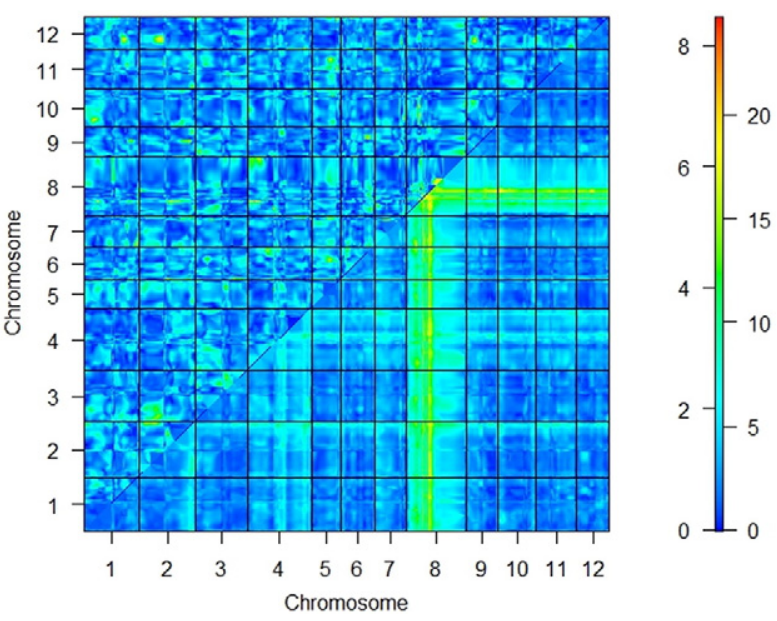

Fig. 3. Heat map for a two-dimensional genome scan with a two-QTL model. The lower right triangle shows the maximum LOD score for the full model (two QTLs plus an interaction). The upper left triangle displays the maximum LOD score for the interaction model. The values for the interaction model (LOD threshold $=6.5$ ) and full model (LOD threshold $=8$ ) on the left and right, respectively, are shown in a color-coded scale.

enzyme betaine aldehyde dehydrogenase (BADH, EC 1.2.1.8) (Chen et al., 2006). The 'MRQ74' allele of frg8-1 could increase aroma in the population compared with the alleles from the non-fragrant parent. Thus, the fragrance of 'MRQ74' is highly governed by frg8-1. frg8-1 was identified in the same region of chromosome 8 as that observed in previous reports (Ahn et al., 1992; Amarawathi et al., 2008; Lorieux et al., 1996). Because mapping saturated markers in candidate chromosomal regions is required for determining major as well as minor genes controlling aroma in the present study, we added six polymorphic genebased primers to the region surrounding the LOD support interval for the putative QTL on chromosome 8. As a consequence, the fgr locus was limited between RM223 and SCU015RM (82.2-83.82 cM). After mapping and fine-mapping, Chen et al. (2006) successfully limited the fgr gene to a $69 \mathrm{~kb}$ interval as well. Hence, this study not only validated the results of earlier studies but also added important findings to the knowledge of Malaysian fragrant rice.

In addition to a major QTL, another important QTL was found on chromosome 4 at RM5633 with a LOD score of 6.1, and described $3.2 \%$ of the phenotypic variation. The locus determined on chromosome 4 was in line with earlier studies by Lorieux et al. (1996) and Amarawathi et al. (2008) that showed only little differences in its genetic position. According to findings in the rice genome database for annotated functional genes, badh1 is a potential candidate gene for the fragrance QTL frg4-1 because of its similarity to the badh2 gene on chromosome 8 . BADH1 is involved in stress tolerance, but its function in fragrance has not yet been verified (Bradbury et al., 2005a), while its function is similar to that of the badh2 gene (Amarawathi et al., 2008). To identify epistatic interactions between the QTLs, we used a two-dimensional scan; however, no important epistatic interactions were found in the genome in this population.

In contrast with the idea that segregation distortion loci (SDL) are typically detrimental to QTL mapping, QTL mapping has the potential to benefit from SDL (Xu, 2008). Based on the study by Wang et al. (2005), genomic regions with severe SD equally contain QTLs, and more QTLs will be missed with the deletion of markers in such areas. Thus, these authors suggested application of the adjusted marker map after inserting distorted markers. This approach is capable of recovering QTLs included in the segregation-distorted areas of the genome. From another perspective, if the SDL is present but ignored when QTL mapping is performed, the power will show only a slight decrease. In addition, the power loss is negligible when the marker map is dense because the distorted proportions of QTL genotypes only impact the prior probability of a QTL genotype. The prior probability is less significant in assessing the conditional probability providing marker information. Thus, as a significant consequence, the classical approach of QTL mapping can be used safely without concern for SDL presence. Furthermore, the impact of SDL becomes irrelevant when the genotype of a marker is observed. Therefore, the same formula (conditional probability) can be applied to distorted and undistorted markers (Xu, 2008). In fact, appropriate management of distorted markers not only decreases their impact on genetic map construction and QTL mapping (Alheit et al., 2011; Xu and Hu, 2009) but may also be useful for QTL mapping (Alheit et al., 2011; Xu, 2008). Hence, in this study, the impact of SDLs is irrelevant due to the quality filtering of the used markers, observable genotypes of the markers, dense marker map, and no linkage between the QTLs and distorted markers. Apart from these important issues, SDLs are harmful to the power of dominance QTL detection, while both detected QTLs in this study were additive.

Apparently, there are contrasting observations with regard to the aroma gene and the nature of inheritance, and the number of genes and genetic loci involved (Sakthivel et al., 2009b) has shown the probability of control through different rice fragrance genes (either dominant or recessive). These probabilities include the following: one main QTL on chromosome 8 and two minor QTLs on chromosomes 3 and 4 (Amarawathi et al., 2008), two to three recessive or dominant genes (Reddy and Reddy, 1987), two recessive genes (Hien et al., 2006), one major QTL located on chromosome 8 and two minor QTLs on chromosomes 4 and 12 (Lorieux et al., 1996), a single dominant gene (Kuo et al., 2005), a dominant suppression epistasis interaction between two genes (Chaut et al., 2010) and an interaction between two genes (complimentary gene action) (Chaut et al., 2010). The genetic analyses in the present research also revealed that the aroma trait in the mapping population showing quantitative inheritance was controlled by a combination of multiple loci with major and minor actions.

To further explain the evolution and molecular aspects resulting in the influential exploitation of fragrant rice, progressive metabolomics and genomic approaches, comparative mapping and exploration of the expression levels of differentially regulated genes between aroma and non-aroma lines are necessary (Golestan Hashemi et al., 2013). Moreover, chromosome segment substitution lines appear to be useful instruments for identifying minor QTLs since they can separate minor QTLs from major QTLs situated in other chromosome regions. To confirm the actual co-segregation between candidate genes and trait variation, the QTLs gained from this study should be validated in additional progenies as well as environments to increase the reliability and power of QTL detection together with gene expression studies and association mapping (Jeennor and Volkaert, 2014). The significant QTLs influencing fragrant grain quality found in the present study can be utilized by breeders for crop improvement plans and the further fine mapping of particular genes to enhance gene-based perfect markers for use in rice breeding and the mining of better alleles for these genes in aromatic rice collections. Consequently, for better comprehension, genetic and environmental criteria that might impact this prominent characteristic should be taken into account.

\section{Conflict of interest}

The authors declare no conflict of interest.

\section{Acknowledgments}

The authors express their acknowledgements of the Long-term Research Grant Scheme (LRGS) (5525001), Food Security Project, Ministry of Higher Education, Malaysia, for the financial support to conduct activities on rice research program. 


\section{References}

Ahn, S., et al., 1992. RFLP tagging of a gene for aroma in rice. Theor. Appl. Genet. 84, 825-828.

Alheit, K.V., et al., 2011. Detection of segregation distortion loci in triticale ( $\times$ Triticosecale Wittmack) based on a high-density DArT marker consensus genetic linkage map. BMC Genomics 12, 380.

Amarawathi, Y., et al., 2008. Mapping of quantitative trait loci for basmati quality traits in rice (Oryza sativa L.). Mol. Breed. 21, 49-65.

Bradbury, L.M., et al., 2005a. The gene for fragrance in rice. Plant Biotechnol. J. 3, 363-370.

Bradbury, L.M., et al., 2005b. A perfect marker for fragrance genotyping in rice. Mol. Breed. $16,279-283$.

Broman, K.W., et al., 2003. R/qtl: QTL mapping in experimental crosses. Bioinformatics 19 889-890.

Chaut, A.T., et al., 2010. Genetic analysis for the fragrance of aromatic rice varieties. 3rd International Rice Congress, November 8-12, Hanoi, Vietnam, p. 3898.

Chen, S., et al., 2006. The fgr gene responsible for rice fragrance was restricted within 69 kb. Plant Sci. 171, 505-514.

Childs, N., Burdett, A., 2000. The US Rice Export Market Rice Situation and Outlook, USDAERS, November, RCS-2000.

Cordeiro, G.M., et al., 2002. Identification of microsatellite markers for fragrance in rice by analysis of the rice genome sequence. Mol. Breed. 9, 245-250.

Gawel, N., Jarret, R., 1991. A modified CTAB DNA extraction procedure for Musa and Ipomoea. Plant Mol. Biol. Report. 9, 262-266.

Golestan Hashemi, F.S., et al., 2013. Biochemical, genetic and molecular advances of fragrance characteristics in rice. Crit. Rev. Plant Sci. 32, 445-457.

Hien, N., et al., 2006. Evaluation of Aroma in Rice (Oryza sativa L.) using KOH Method Molecular Markers and Measurement of 2-Acetyl-1-Pyrroline Concentration. Nettai nogyo Jpn. J. Tropical Agric. 50, 190-198.

Huang, F., et al., 1998. Present situations and prospects for the research on rice grain quality forming. Chin. J. Rice Sci. 12, 172-176.

Jeennor, S., Volkaert, H., 2014. Mapping of quantitative trait loci (QTLs) for oil yield using SSRs and gene-based markers in African oil palm (Elaeis guineensis Jacq.). Tree Genet Genomes 10, 1-14.

Khush, G.S., Brar, D., Hardy, B., 2001. Rice Genetics IV. Science Publishers, USA.

Kosambi, D., 1943. The estimation of map distances from recombination values. Ann. Eugenics $12,172-175$

Kovach, M.J., et al., 2009. The origin and evolution of fragrance in rice (Oryza sativa L.) Proc. Natl. Acad. Sci. 106, 14444-14449.
Kuo, S.M., et al 2005. The betaine aldehyde dehydrogenase ( $B A D 2$ ) gene is not responsible for aroma trait of AS0420 rice mutant derived by sodium azide mutagenesis. Proceedings of the 5th International Rice Genetics Symposium. IRRI, Philippines.

Lorieux, M., et al., 1996. Aroma in rice: genetic analysis of a quantitative trait. Theor. Appl. Genet. 93, 1145-1151.

McCouch, S.R., 2008. Gene nomenclature system for rice. Rice 1, 72-84.

McCouch, S.R., et al., 2002. Development and mapping of 2240 new SSR markers for rice (Oryza sativa L.). DNA Res. 9, 257.

Pinson, S.R.M., 1994. Inheritance of aroma in six rice cultivars. Crop Sci. 34, 1151-1157.

R Core Team, 2011. R: A Language and Environment for Statistical Computing. R Foundation for Statistical Computing, Vienna, Austria (2012 Open access available at: http:// cranr-projectorg)

Reddy, V., Reddy, G., 1987. Genetic and biochemical basis of scent in rice (Oryza sativa L.). Theor. Appl. Genet. 73, 699-700.

Saha, P.S., et al., 2012. Molecular characterization of aromatic Oryza sativa L. cultivars from West Bengal, India. Nucleus 55, 83-88.

Sakthivel, K., et al., 2009a. Development of a simple functional marker for fragrance in rice and its validation in Indian Basmati and non-Basmati fragrant rice varieties. Mol. Breed. 24, 185-190.

Sakthivel, K., et al., 2009b. Genetic and molecular basis of fragrance in rice. Biotechnol. Adv. 27, 468-473.

Shi, W., et al., 2008. Discovery of a new fragrance allele and the development of functional markers for the breeding of fragrant rice varieties. Mol. Breed. 22, 185-192.

Sun, S.X., et al., 2008. Genetic analysis and gene fine mapping of aroma in rice (Oryza sativa L. Cyperales, Poaceae). Genet. Mol. Biol. 31, 532-538.

Temnykh, S., et al., 2000. Mapping and genome organization of microsatellite sequences in rice (Oryza sativa L.). Theor. Appl. Genet. 100, 697-712.

Vanavichit, A., Yoshihashi, T., 2010. Molecular aspects of fragrance and aroma in rice. In: Kader, J.C., Delseny, M. (Eds.), Advances in Botanical Research vol. 56. Elsevier, pp. 49-73.

Wang, C., et al., 2005. Mapping segregation distortion loci and quantitative trait loci for spikelet sterility in rice (Oryza sativa L.). Genet. Res. 86, 97-106.

$\mathrm{Xu}, \mathrm{S}$. , 2008. Quantitative trait locus mapping can benefit from segregation distortion. Genetics 180, 2201-2208.

$\mathrm{Xu}, \mathrm{S}$., Hu, Z., 2009. Mapping quantitative trait loci using distorted markers. Int. J. Plant Genomics 2009, 1-11.

Zhang, H., et al., 2013. RCircos: an R package for Circos 2D track plots. BMC Bioinforma. 14, 244. 